\title{
PRÁTICAS DE AUTOCUIDADO EM SAÚDE MENTAL EM CONTEXTO DE PANDEMIA
}

\author{
Elizabeth Esperidião' \\ ORCID: 0000-0002-9284-6243
}

Marciana Gonçalves Farinhas"

ORCID: 0000-0002-2024-7727

Maria Giovana Borges Saide I"' ORCID: 0000-0002-3259-1760

'Faculdade de Enfermagem, Universidade Federal de Goiás. Goiânia, Goiás, Brasil.

"Instituto de Psicologia, Universidade Federal de Uberlândia. Uberlândia, Minas Gerais, Brasil.

I"'Faculdade de Enfermagem, Universidade Estadual de Campinas. Campinas, São Paulo, Brasil.

Autor Correspondente: Elizabeth Esperidião E-mail: betesper@ufg.br

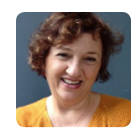

Como citar:

Esperidião E, Farinhas MG, Saidel MGB. Práticas de autocuidado em saúde mental em contexto de pandemia. In: Esperidião E, Saidel MGB (Orgs.). Enfermagem em saúde mental e COVID-19. 2.ed. rev. Brasília, DF: Editora ABEn; 2020.

p. 65-71. (Série Enfermagem e Pandemias, 4). https://doi.org/10.51234/aben.20.e04.c09

\section{INTRODUÇÃO}

A pandemia da COVID-19, declarada pela Organização Mundial de Saúde em março de 2020, configura-se como uma situação de crise. A síndrome caracterizada por um conjunto de doenças respiratórias agudas foi identificada em dezembro de 2019 em Wuhan, República Popular da China ${ }^{(1)}$. A partir de então, o cenário mundial tem sido transformado pelos impactos políticos, econômicos, sociais e culturais que a pandemia apresenta nos países atingidos.

No Brasil, diversas medidas foram tomadas para o enfrentamento da emergência de saúde, tais como: recomendações de distanciamento social; isolamento; quarentena ou bloqueio total, chamado também de lockdown. Este contexto novo, imposto no cotidiano das pessoas abruptamente, afetou rotinas e a configuração das relações interpessoais, no âmbito social, familiar e ocupacional, repercutindo, inevitavelmente, na saúde mental dos indivíduos. Restrições, o medo e a ansiedade relacionada à doença podem ser intensos e causar impactos psicológicos negativos na população(2). Pesquisas recentes descrevem a relação de sintomas de sofrimentos psíquico com a pandemia de COVID-19 e fazem considerações sobre a saúde mental e a pandemia(3-4).

Assim, para além da epidemiologia, tratamento e manejo da doença, as implicações da saúde mental precisam ser consideradas diante do impacto que a COVID-19 tem trazido na saúde mental e qualidade de vida das pessoas. Pesquisadores alertam para a necessidade de incluir medidas protetivas de saúde mental nos protocolos de atendimento à comunidade e aos profissionais de saúde ${ }^{(3,5)}$. Essas ações protetivas podem oferecer cuidado em diferentes dimensões como a individual e a coletiva que minimizem os efeitos da pandemia na vida das pessoas.

Há também impactos na saúde mental dos profissionais de saúde que precisam ser considerados, visando oferta de assistência especializada em saúde mental a fim de mitigar o sofrimento psíquico durante e pós pandemia. Embora os 
profissionais de saúde estejam acostumados e tenham competência para tomar deliberações de vida e morte nas suas rotinas de trabalho, o contexto da pandemia traz mais insegurança quanto à oferta suficiente de suprimentos e equipamentos fundamentais para a realização de suas práticas de cuidado, exigindo-lhes muitas vezes a decisão de selecionar qual paciente precisa mais urgentemente de determinados instrumentos ${ }^{(3,5)}$.

Abordar e refletir as muitas questões atreladas ao sofrimento psíquico, muitas vezes invisíveis, embora não menos importantes, é premente, pois estamos vivendo a pandemia do medo, que causa angústia e muito desconforto emocional nas pessoas. Podemos não ver o que nos inquieta, mas sentimos muito intensamente.

\section{OBJETIVO}

Refletir e propor práticas de autocuidado em saúde mental para situações de pandemia.

\section{MÉTODO}

O capítulo foi construído na perspectiva teórico-reflexiva de ações e possibilidades sobre o autocuidado em saúde mental, especialmente em tempos de pandemia. A edificação fundamentou-se a partir da leitura crítica de referências nacionais e internacionais que abordam o tema, diante da crise sanitária decorrente da COVID-19, em todo o mundo, além de considerações pautadas na experiência das autoras na área de saúde mental. As reflexões apresentadas foram categorizadas em tópicos que indicam os impactos psicossociais para a saúde mental no contexto em estudo, estratégias de cuidado, além de algumas práticas de autocuidado em saúde mental.

\section{RESULTADOS}

\section{Impactos psicossociais da pandemia junto a população em geral}

A forma como as pessoas enfrentam as adversidades do momento de limitações e restrições é subjetiva e particular, consequentemente o impacto na saúde mental também. Ainda que a grande maioria da população esteja sendo impactada pela pandemia, é preciso considerar que podemos sair mais fortalecidos desta crise sanitária com repercussões positivas na nossa saúde mental.

Os impactos são, invariavelmente, de diversas naturezas, desde os psicossociais, que envolvem também as necessidades gregárias, com todo o universo das relações interpessoais, de segurança e estabilidade financeira às necessidades psicobiológicas, as quais influenciam diretamente o nível de satisfação e realização do ser humano. Na medida em que alguma das necessidades básicas não for atendida, pode-se comprometer o bem-estar e equilíbrio da saúde física e mental.

No cenário da pandemia as restrições na liberdade de ir e vir, em compartilhar presencialmente afetos, vontade de estar perto, de ver, sentir, tocar, se constituem em ameaças e deixam as pessoas mais vulneráveis e fragilizadas ${ }^{(5,6)}$. As repercussões econômicas decorrentes das medidas adotadas por grande parte dos municípios brasileiros atingidos são igualmente responsáveis pela equilibração emocional da população(7). Diante da diversidade socioeconômica brasileira, os cidadãos estão enfrentando desigualmente esse período: restrições não apenas do contato físico, mas de tantas outras necessidades básicas, de sobrevivência inclusive.

Estamos numa conjuntura de epidemia oculta, no qual a insegurança e receios vão se apropriando das pessoas, tornando o comprometimento da saúde mental quase inevitável. O desafio é, portanto, encontrar estratégias de superação das dificuldades para o alcance do bem estar aliado ao movimento de (re)significar as experiências para transformá-las em impactos positivos.

O distanciamento social tem proporcionado a aproximação familiar, embora nem sempre de forma saudável, afetiva ou prazerosa. Muitos de nós temos pessoas por perto que podemos contar, mas há quem tem por perto pessoas que, ao contrário, podem atrapalhar. Já existem dados do aumento da violência doméstica, violência 
à mulher, do uso abusivo de álcool e outras drogas, incluindo medicamentos psicoativos como forma de superação ou evitação do problema ${ }^{(8-9)}$. Neste panorama evidencia-se sinais de sofrimento psíquico para muitos e ou o agravamento de transtornos mentais naqueles com histórico pregresso de episódios psiquiátricos.

Na população em geral várias são as queixas, pedidos de ajuda, muitos relatos de exaustão mental, de apatia, quadros de ansiedade, insônia, pesadelos, irritabilidade, baixa produção, dificuldade de concentração, compulsão alimentar, dilemas e dificuldades de toda ordem no enfrentamento das atividades cotidianas(4). Por exemplo, dar conta de estudar ou participar de reuniões utilizando ferramentas remotas, acompanhar lives de atualização profissional, exigem muito mais concentração. O trabalho em home office, acompanhar os filhos em homeschooling, gerenciar tarefas domésticas, reforço nas medidas de proteção corporal, passaram a fazer parte do dia a dia de grande parcela da população, entre tantas outras atividades significativamente diferentes das usuais, exigindo maior esforço de adaptação.

Essas atividades cotidianas com o passar do tempo e a imprevisibilidade da situação futura, podem corroborar para intensificação de sentimentos de sofrimento psíquico. Tais sentimentos precisam ser considerados como incapacidades naturais diante do contexto pandêmico, e os indivíduos que estão vivenciando essa realidade, necessitam serem alvos de cuidados e orientações sobre saúde mental.

\section{Impactos psicossociais da pandemia junto à profissionais de saúde}

Entre os profissionais de saúde, é comum manifestações de medo de contaminarem seus familiares e a insegurança quanto à proteção adequada com os equipamentos de proteção individual (EPI) disponibilizados para o uso. Há ainda o medo de adoecer que pode denunciar medo da morte, de deixar a equipe sobrecarregada ou ainda culpa por sobreviver diante de tantas mortes. Ficam impactados emocionalmente por assistirem em pouco tempo perdas de pacientes que não conseguiram salvar ${ }^{(3)}$. Estudo com equipes de saúde após surto do Ebola, encontrou sintomas de desgaste, isolamento social e transtorno de estresse pós-traumático ${ }^{(10)}$. As epidemias e surtos de contaminação de doenças geralmente são seguidos de impactos psicossociais individuais e sociais de grande monta ${ }^{(5)}$

Por tais razões há iniciativas de atendimento em saúde mental online, protagonizadas por especialistas enfermeiros e psicólogos, algumas mediadas pelos conselhos de classe ou por instituições que tenham alcancem aos seus profissionais. Essas iniciativas já vivenciadas em outros países, que hoje estão em outro estágio da pandemia, têm demonstrado bons resultados relacionados ao acolhimento e possíveis intervenções para esse público ${ }^{(2,4)}$.

Os relatos de profissionais de enfermagem atendidos incluem: - ansiedade, em razão da falta de EPIs, pressão da chefia imediata, notícias da mídia sobre a doença, o número de mortes nos plantões; - medo de se infectar e o risco de transmitir o vírus aos seus familiares; - ambivalência, pois por um lado as pessoas valorizam o seu trabalho e por outro os discriminam não querendo contato com eles; - tristeza e queixas depressivas, associadas à solidão, afastamento das famílias, morte de profissionais da equipe de trabalho, estarem vivendo e acompanhando a fragilidade do sistema hospitalar; e a exaustão ou esgotamento emocional, decorrente do volume de trabalho aumentado nesse período pandêmico ${ }^{(2-3)}$. A longo prazo essas circunstâncias aumentam o risco de desenvolvimento de transtornos de estresse pós-traumático (TEPT), depressão, outros transtornos de ansiedade, uso indevido de substâncias e suicídio(11).

\section{Estratégias de cuidado em saúde mental}

São várias as possibilidades de ações voltadas ao cuidado da saúde mental. Incluem-se medidas protetivas protagonizadas por especialistas, por gestores ou pela própria pessoa, que podem empreender algumas delas em prol do seu bem-estar, mesmo diante das limitações impostas pelo cenário pandêmico. Considerando que os serviços especializados de atenção psicossocial são insuficientes ou nem sempre acessíveis à maior parte da população, investir em orientações para práticas de autocuidado deveria ser uma prioridade nos atendimentos em saúde. 
Profissionais especialistas em saúde mental têm procurado atender a grande demanda ora instalada em razão da pandemia pelo COVID-19. As atividades são diversas, desde as contribuições para a ciência, participando em editais de pesquisa, publicando artigos científicos, elaborando protocolos de atendimento, promovendo capacitações, além dos atendimentos de acolhimento e escuta terapêutica.

De igual maneira, sabe-se do engajamento dos profissionais psicólogos no suporte emocional de servidores dos serviços de saúde ou de instituições de ensino, direcionado ao corpo docente e discente, para além do atendimento psicoterápico ${ }^{(12)}$.

No Brasil, existe um canal de atendimento online exclusivo ao apoio à saúde mental de profissionais de Enfermagem, em virtude do COVID-19, coordenado pelo Conselho Federal de Enfermagem. É um serviço 24 horas, em que enfermeiros especialistas em saúde mental, de todo o Brasil, se disponibilizam voluntariamente para acolher e dar suporte emocional aos que sentirem necessidade de compartilhar suas aflições. Neste espaço de escuta, observa-se as mesmas queixas da população em geral, somadas aos riscos decorrentes do exercício profissional, que os coloca em condições de maior vulnerabilidade ${ }^{(13)}$.

\section{Práticas de autocuidado em saúde mental}

Algumas medidas protetivas de autocuidado estão dispostas neste capítulo mais no sentido de oferecer possibilidades para cada um encontrar alívio do desconforto emocional ou ainda servir como sugestões na planificação de cuidados dos profissionais às pessoas que procurarem por ajuda.

Neste sentido, as práticas de autocuidado em saúde mental podem beneficiar as pessoas, quando empreendidas e incorporadas sistematicamente no cotidiano, pois se configuram como medidas de promoção à saúde. Momentos de crise podem ser tempos de resgate e se constituírem em oportunidades de aprendizado e crescimento pessoal. É preciso considerar que, mesmo com os infortúnios psicossociais decorrentes da pandemia, é possível o alcance de momentos de bem-estar.

Num estudo realizado na Irlanda com 604 pessoas de ambos os sexos, após o fechamento de escolas e serviços não essenciais, durante o período de distanciamento social mostrou que atividades ao ar livre como exercitar, atividades ligadas à terra, se dedicar a hobbies, contribuíram para diminuir as emoções negativas das pessoas, sugerindo o papel protetor relacionado ao bem-estar durante esse momento de pandemia ${ }^{(14)}$. Há de se resgatar atitudes simples para o bem viver, buscando mecanismos de auto suporte no contraponto das inquietações e receios vivenciados. Reconhecer e acolher as próprias limitações, ainda que possa trazer desconforto, é sem dúvida um bom começo e absolutamente saudável. Recomenda-se avaliar novas e ou conhecidas estratégias exitosas do próprio repertório existencial em situações de dificuldades com vistas a implementá-las. O movimento de introspecção, voltado ao autoconhecimento deve ser encorajado, para (re)descobrir o que é ou pode ser prazeroso no cotidiano, a despeito das mudanças na rotina em função do momento das restrições psicossociais.

As práticas de Mindfulness, por exemplo, incentivam que voltemos a atenção plena ao momento presente e autocompaixão com experiências desafiadoras, buscando perceber pensamentos, sensações corporais e emoções no momento que ocorrem, aceitando-os sem reagir de maneira habitual. Em todo o mundo encontram-se estudos que comprovam os inúmeros dos seus benefícios para a saúde física e mental ${ }^{(15)}$

De igual forma, as práticas integrativas e complementares em saúde, reconhecidas em suas ações junto ao Sistema Único de Saúde brasileiro, têm contribuído na promoção de saúde, qualidade de vida e equilíbrio entre mente, corpo e espírito. Existem grupos de especialistas que publicaram cartilhas com sugestões e orientações de várias práticas para o autocuidado, especialmente úteis para enfrentar os efeitos psíquicos da pandemia ${ }^{(16)}$. Iniciativas que visem o alívio de tensões em momentos de desajustes emocionais são altamente indicadas e resolutivas para o equilíbrio e cuidado do sofrimento psíquico, influenciando positivamente a saúde mental.

Promover momentos de pausa ao longo do dia, para se perceber, respirar lenta e profundamente trazendo boa energia para o corpo e eliminando sensações desagradáveis são medidas igualmente saudáveis e desejáveis no cotidiano. 
Refletir sobre preferências e prazeres. Como estão sendo atendidos? Listar o que se gosta de fazer pode ser um exercício apropriado para dar visibilidade às necessidades que estão sendo satisfeitas para, numa próxima etapa, redirecionar algumas ações de autocuidado, se for o caso. Este exercício favorece também a identificação do que não é prazeroso e, na medida do possível, pode-se substituir por iniciativas que ofereçam mais sensações agradáveis.

Encontrar novos prazeres, resgatar alguns esquecidos: proximidade com a música, filmes, prazer em cozinhar, organizar a casa, cuidar de plantas, animais, do carro, aprender ou desenvolver idiomas, visitar sites, museus e serviços de entretenimento, cursos online. Atualmente com a internet várias são as opções disponíveis e acessíveis sem a necessidade de muito aparato tecnológico. De igual forma, é desejável incorporar práticas meditativas, contemplação da natureza, exercícios de respiração, de relaxamento que ajudam no equilíbrio físico e mental, além da aproximação com a religiosidade, favorecem conforto e benefícios na redução de estresse, insônia e paz interior.

Seguir a rotina mais próxima possível da usual, tentando manter os hábitos: hora do trabalho, do estudo, do exercício físico, do sono, do trabalho doméstico, da rotina das compras, acompanhamento atividades escolares dos filhos. Mesmo em home office, manter-se arrumado favorece o bem-estar, autoestima.

Saborear mais os afazeres, empreender com leveza as atribuições e compromissos no trabalho e familiares. Dar espaço para a alimentação saudável, hidratação, exposição ao sol.

Investir em ações compartilhadas de cuidado e de solidariedade: aumenta a sensação de pertença social. Manter ativa a rede sócio afetiva: participar, organizar encontros virtuais com pessoas significativas. Extrapolar para além das mensagens de textos, que não expressam tom de voz e não favorecem o fortalecimento de vínculo. Ainda que o panorama da pandemia apresente muitas adversidades vivenciadas pelo distanciamento social, percebe-se uma gama de possibilidades da proximidade afetiva, mediadas especialmente pelos recursos da tecnologia digital.

Dieta de notícias: filtrar e se informar em fontes seguras, sem exageros. Divulgar histórias positivas e imagens de pessoas que se recuperaram da doença, são meios de instilar esperança e minimizar sensações de medos e inseguranças.

Procurar ajuda profissional diante de fragilidades não superadas com as atitudes de autocuidado é uma importante e potente possibilidade de atenção para consigo próprio. Em tempos em que o isolamento social está instalado, existem profissionais e serviços que estão sendo disponibilizados por meios de ferramentas remotas para o atendimento de necessidades relacionas à estabilização emocional.

\section{CONSIDERAÇÕES FINAIS}

As práticas de autocuidado são essenciais para o enfrentamento de situações de vulnerabilidade, além de serem consideradas medidas de prevenção do adoecimento psíquico. A literatura apontou possibilidades de ações e estratégias protetivas em saúde mental a serem empreendidas no cotidiano, no sentido aliviar o sofrimento psíquico e o agravamento de sintomas de transtornos mentais pré-existentes.

As práticas de autocuidado são essenciais para o enfrentamento de situações de vulnerabilidade e dos diferentes impactos decorrentes da pandemia, tanto para a população em geral, quanto para os profissionais de saúde. A experiência de vários países que vem enfrentando os dilemas e implicações da grave crise sanitária pelo COVID-19, tem sido retratada pela literatura que sinaliza inúmeras possibilidades de ações a serem empreendidas no dia a dia, no sentido de prevenir o adoecimento psíquico, bem como a agudização de transtornos mentais pré-existentes. Práticas de autocuidado contribuem significativamente para a ampliação de autoconhecimento, além de desenvolver a maturidade psíquica bem como os processos de resiliência. Importante serem incorporadas sistematicamente no cotidiano das pessoas, pois a periodicidade, frequência e intensidade, são fundamentais para o equilíbrio e bem-estar almejado.

É consenso entre os organismos nacionais, internacionais e pesquisadores a relevância em incluir medidas protetivas de saúde mental nos protocolos de atendimento não somente aos profissionais de saúde, mas à comunidade em geral, considerando que os impactos psicossociais poderão persistir em tempos de pós 
pandemia. Há de se destacar a recomendação do acompanhamento por especialistas da área da saúde mental nas situações em que o indivíduo apresente sintomas de sofrimentos psíquico. Por fim, vale dizer que as medidas apresentadas nesse capítulo se configuram sob a égide da promoção da saúde mental, na busca da melhor qualidade de vida das pessoas.

\section{Limitações do Estudo}

Dentre as limitações do estudo têm-se as pesquisas realizadas com países de climas, culturas e comportamentos diferentes do que o Brasil o que denuncia a importância de pesquisas nacionais sobre a temática que nos possibilite ampliar a compreensão do fenômeno. Trata-se de um estudo teórico reflexivo que sintetiza o que foi pensado para a realidade até o momento podendo não dar conta da evolução do fenômeno daqui para a frente com outras emergências surgindo.

\section{Contribuições para a Área}

O texto aborda inúmeras estratégias que podem contribuir para que as pessoas ampliem o repertório existencial e se instrumentalizem no que diz respeito às práticas de autocuidado para a promoção e proteção de sua saúde mental. Essas estratégias podem contribuir tanto para o contexto atual, como para outras situações pandêmicas que poderão impor igualmente o isolamento social como necessidade. Além disso, vários segmentos da população podem ser beneficiados com as ações e estratégias abordadas, ao serem colocadas em prática, nos serviços de saúde por equipes multidisciplinares, na perspectiva da educação permanente e continuada.

\section{AGRADECIMENTO}

Ao Departamento de Enfermagem Psiquiátrica e Saúde Mental/Associação Brasileira de Enfermagem (DEPSM/ABEn).

\section{REFERÊNCIAS}

1. World Health Organization (WHO). Coronavirus disease 2019 (COVID-19): situation report - 78. [Internet] 2020 [cited 2020 jun 15] Geneva. Available from: http://www.who.int/docs/default-source/coronaviruse/situation-reports/20200407-sitrep78-covid-19.pdf?sfvrsn=bc43e1b_2

2. Brooks SK, Webster RK, Smith LE et al. The psychological impact of quarantine and how to reduce it: rapid review of the evidence. The Lancet. [Internet] 2020 [cited 2020 jun 22];395(102227),912-920. Available from: https://www.thelancet. com/journals/lancet/article/PIIS0140-6736(20)30460-8/fulltext

3. Lai J, Ma S, Wang Y, et al. Factors Associated With Mental Health Outcomes Among Health Care Workers Exposed to Coronavirus Disease 2019. JAMA Netw Open. [Internet] 2020 [cited 2020 jun 23];3(3):e203976. Available from: https:// jamanetwork.com/journals/jamanetworkopen/fullarticle/2763229

4. Wang C, Pan R, Wan X et al. Immediate psychological responses and associated factors during the initial stage of Coronavirus Disease (COVID-19) epidemic among the general population in China. Int J Environ Res Public Health [Internet] 2020 [cited 2020 jun 23];17(5),1729. Available from: https://www.ncbi.nlm.nih.gov/pmc/articles/PMC7084952/

5. Duan L, Zhu G. Psychological interventions for people affected by the COVID-19 epidemic. The Lancet. [Internet] 2020 [cited 2020 jun 24];7 300-302. Available from: https://www.ncbi.nlm.nih.gov/pmc/articles/PMC7128328/

6. Encarnação P, Macedo E, Machado M et al. Efeito do Toque Terapêutico na Dor, Autocuidado, Depressão e Cortisol: Estudo de um Caso Clínico. Revista De Investigação \& Inovação Em Saúde. [Internet] 2018 [cited 2020 jun 24];1(1),17-24. Available from: https://doi.org/10.37914/riis.v1i1.25

7. Silva JB, Muniz AMV. 2020. Pandemia do Coronavírus no Brasil: Impactos no Território Cearense, Espaço e Economia [Internet] 2020 [citado 202030 jun]; 17, Available from: https://doi.org/10.4000/espacoeconomia.10501 
8. Telles LEB, Valença AM, Barros AJS et al. Domestic violence in the COVID-19 pandemic: a forensic psychiatric perspective. Braz. J. Psychiatry. [Internet] 2020 [cited 2020 jun 25]. Available from: https://www.scielo.br/scielo. php?pid=S1516-44462020005015211\&script=sci_arttext

9. World Health Organization (WHO). COVID-19 and violence against women: what the health sector/system can do. [Internet] 2020 [cited 2020 jun 25] Geneva. Available from: https://www.who.int/reproductivehealth/publications/vaw-covid-19/en/

10. Paladino L, Sharpe RP, Galwankar SC et al. Reflections on the Ebola public health emergency of international concern, part 2: The unseen epidemic of posttraumatic stress among health-care personnel and survivors of the 2014-2016 Ebola outbreak. J Global Infect Dis [Internet] 2017 [cited 2020 jun 25];9:45-50. Available from: http://www.jgid.org/text.asp?2017/9/2/45/206945

11. Li Z, Ge J, Yang M, et al. Vicarious traumatization in the general public, members, and non-members of medical teams aiding in COVID-19 control. Brain Behav Immun. [Internet] 2020 [cited 2020 jun 27];S0889-1591(20)30309-3. Available from: https://www.ncbi.nlm.nih.gov/pmc/articles/PMC7102670/

12. Conselho Federal de Psicologia (Brasil). Resolução do exercício profissional n4, de 26 de março de 2020. Dispõe sobre regulamentação de serviços psicológico prestados por meio de Tecnologia da Informação e da Comunicação durante a pandemia do COVID19. [Internet] 2020 [cited 2020 jun 29]. Available from: https://atosoficiais.com.br/cfp/resolucaodo-exercicio-profissional-n-4-2020-dispoe-sobre-regulamentacao-de-servicos-psicologicos-prestados-por-meio-detecnologia-da-informacao-e-da-comunicacao-durante-a-pandemia-do-covid19?origin=instituicao

13. Humerez DC, OhI RIB, Silva MCN. Saúde Mental dos profissionais de enfermagem do Brasil no contexto da Pandemia COVID-19: Ação do Conselho Federal de Enfermagem. Cogitare Enfermagem. [Internet] 2020 [cited 2020 jun 27];25:21769133. Available from: https://revistas.ufpr.br/cogitare/article/view/74115

14. Lades LK, Laffan K, Daly M et al. Daily emotional well-being during the COVID-19 pandemic. Br. J. Health Psychol. [Internet] 2020 [cited 2020 jun 29]. Available from: https://onlinelibrary.wiley.com/doi/full/10.1111/bjhp.12450

15. Behan C. The benefits of meditation and mindfulness practices during times of crisis such as COVID-19. Irish Journal of Psychological Medicine. [Internet] 2020 [cited 2020 jun 30];1-3 Available from: https://doi.org/10.1017/ipm.2020.38

16. Nelson ICASR (org). Cartilha Ser-Mente: inserindo práticas integrativas no seu dia a dia. [Internet] 2020 [cited 2020 jun 30 ]; Mossoró RN: EDUERN. Available from: http://www.uern.br/pdf/Documentos/CartilhaSERMENTE.pdf 